\title{
The association between chronic airflow obstruction and poverty in 12 sites of the multinational BOLD study
}

\author{
John Townend ${ }^{1}$, Cosetta Minelli ${ }^{1}$, Kevin Mortimer $^{2}$, Daniel O. Obaseki ${ }^{3}$, \\ Mohammed Al Ghobain ${ }^{4}$, Hamid Cherkaski ${ }^{5}$, Myriam Denguezli, \\ Kirthi Gunesekera ${ }^{7,8}$, Hasan Hafizi ${ }^{9}$, Parvaiz A. Koul ${ }^{10}$, Li C. Loh ${ }^{11}$, \\ Chakib Nejjari $^{12}$, Jaymini Patel ${ }^{1}$, Talant Sooronbayev ${ }^{13}$, Sonia A. Buist ${ }^{14}$ and \\ Peter G.J. Burney ${ }^{1}$
}

\begin{abstract}
Affiliations: ${ }^{1}$ National Heart and Lung Institute, Imperial College, London, UK. ${ }^{2}$ Liverpool School of Tropical Medicine, Liverpool, UK. ${ }^{3}$ Dept of Medicine, Obafemi Awolowo University, Ile-Ife, Nigeria. ${ }^{4}$ Dept of Medicine, College of Medicine, King Saud bin Abdulaziz University for Health Sciences, Riyadh, Saudi Arabia. ${ }^{5}$ Dept of Pneumology, Faculty of Medicine Annaba, University Badji Mokhtar of Annaba, Annaba, Algeria. ${ }^{6}$ Faculté de Médecine Dentaire de Monastir, Université de Monastir, Département des Sciences Fondamentales et Mixtes, Laboratoire de Physiologie et des Explorations Fonctionnelles, Faculté de Médecine de Sousse, Université de Sousse, Sousse, Tunisia. ${ }^{7}$ Central Chest Clinic, Colombo, Sri Lanka. ${ }^{8}$ The National Hospital of Sri Lanka, E W Perera Mawatha, Colombo, Sri Lanka. ${ }^{9}$ Third Pulmonology Service, Faculty of Medicine, Tirana University Hospital "Shefqet Ndroqi", Tirana, Albania. ${ }^{10}$ Sher-i-Kashmir Institute of Medical Sciences, Srinagar, India. ${ }^{11}$ Dept of Medicine, Penang Medical College, Penang, Malaysia. ${ }^{12}$ Dept of Epidemiology and Public Health, Faculty of Medicine, University Sidi Mohammed Ben Abdellah, Fez, Morocco. ${ }^{13}$ Pulmonary Division, National Center of Cardiology and Internal Medicine, Bishkek, Kyrgyzstan. ${ }^{14}$ Pulmonary \& Critical Care Medicine, Oregon Health \& Science University, Portland, OR, USA.
\end{abstract}

Correspondence: Peter G.J. Burney, National Heart and Lung Institute, Imperial College, Emmanuel Kaye Building, 1b Manresa Road, London, SW3 6LR, UK. E-mail: p.burney@imperial.ac.uk

@ERSpublications

Poverty is a strong predictor of chronic airflow obstruction independent of age, sex, smoking and tuberculosis http://ow.ly/T2Sz30bdUNC

Cite this article as: Townend J, Minelli C, Mortimer K, et al. The association between chronic airflow obstruction and poverty in 12 sites of the multinational BOLD study. Eur Respir J 2017; 49: 1601880 [https://doi.org/10.1183/13993003.01880-2016].

ABSTRACT Poverty is strongly associated with mortality from COPD, but little is known of its relation to airflow obstruction.

In a cross-sectional study of adults aged $\geqslant 40$ years from 12 sites $(\mathrm{N}=9255)$, participating in the Burden of Obstructive Lung Disease (BOLD) study, poverty was evaluated using a wealth score (0-10) based on household assets. Obstruction, measured as forced expiratory volume in $1 \mathrm{~s}$ (FEV1)/forced vital capacity (FVC) (\%) after administration of $200 \mu \mathrm{g}$ salbutamol, and prevalence of FEV1/FVC<lower limit of normal were tested for association with poverty for each site, and the results were combined by meta-analysis.

Mean wealth scores ranged from 4 in Blantyre (Malawi) and Kashmir (India) to 10 in Riyadh (Saudi Arabia), and the prevalence of obstruction, from 16\% in Kashmir to 3\% in Riyadh and Penang (Malaysia). Following adjustments for age and sex, FEV1/FVC increased by $0.36 \%$ (absolute change) (95\%CI: 0.22 , 0.49; $\mathrm{p}<0.001)$ per unit increase in wealth score. Adjustments for other confounders reduced this effect to $0.23 \%(0.11,0.34)$, but even this value remained highly significant $(\mathrm{p}<0.001)$. Results were consistent across sites $\left(\mathrm{I}^{2}=1 \%\right.$; phet $\left.=0.44\right)$. Mean wealth scores explained $38 \%$ of the variation in mean $\mathrm{FEV} 1 / \mathrm{FVC}$ between sites $\left(\mathrm{r}^{2}=0.385, \mathrm{p}=0.031\right)$.

Airflow obstruction is consistently associated with poverty at individual and community levels across several countries. 


\section{Introduction}

Chronic Obstructive Pulmonary Disease (COPD) is the third most common cause of death in the world [1] and the sixth most common cause of living with disability [2]. National mortality rates from COPD are associated with per capita Gross National Income (GNI) [3]. Changes over time in GNI are also strongly correlated with changes in mortality from COPD [4]. At an individual level, mortality from COPD has been strongly associated with social class in England and Wales [5].

However, little information on the relationship between poverty and the prevalence of airflow obstruction at an individual level is presently available. In the Burden of Obstructive Lung Disease (BOLD) study, an international study of the prevalence of, and risk factors associated with airflow obstruction, an ecological analysis showed very little association between chronic airflow obstruction and GNI [3]. This could have been due to confounding factors at the ecological (country) level, which might have hidden a true association between airflow obstruction and poverty at the individual level. Information at the individual level on the relationship between poverty and spirometry is available from studies that have used a wide variety of indicators of socio-economic status [6]; however, few of these have measured obstruction and those that have done so, provide contradictory results [6]. A French study in adults found an association between obstruction and socioeconomic status [7], whereas a Canadian study among children did not, reporting only an association with lung volumes [8]. Earlier analyses from the BOLD study have shown associations with education, which is associated with wealth $[9,10]$; however, wealth has not yet been assessed at an individual level.

In this study, we examined the association between poverty and airflow obstruction in 12 sites from the BOLD study with an estimated per capita GNI (US\$ purchasing power parity, PPP) ranging between US\$ 750 (Malawi) and US\$ 53760 (Saudi Arabia). Poverty at an individual level was evaluated using a wealth score based on household assets, ranging from 0 (poorest) to 10 (richest). Furthermore, airway obstruction was assessed using post-bronchodilator forced expiratory volume in $1 \mathrm{~s}$ (FEV1)/forced vital capacity (FVC) (\%) and $\mathrm{FEV} 1 / \mathrm{FVC}<$ lower limit of normal (LLN).

\section{Methods}

The BOLD study is an international multi-site study of the prevalence and determinants of chronic lung disease. Details of the study design and methodology have been previously described [11]. Sites were selected in all regions of the world, as defined by the Global Burden of Disease programme, with the exception of Latin America, the Asian Pacific high-income regions and Oceania. Each site was asked to administer questionnaires and carry out spirometric tests on a representative sample of at least 600 adults, aged 40 years and above. All spirometry was conducted using the same equipment and all technicians were trained using the BOLD protocol. Spirometry data were centrally reviewed for quality. Where the quality of any technician fell below the quality required for the programme, the technician was temporarily withdrawn and re-trained. The current analysis included the 12 sites that entered the study after new questionnaires that included the 15 -item asset inventories used to create a measure of individual wealth were introduced [12].

An automated item selection procedure was used to choose a subset of the asset ownership questions, which met the requirements of a Mokken scale [13, 14]. This non-parametric method uses a set of questions that can be ordered in increasing stringency, such that anyone who answers yes to owning one of the assets is likely to have answered yes to all of the more basic assets. Items in our questionnaire were selected because we expected that they would all be desirable, but would cover a range of obtainability such that some would be owned by most people, whilst the less basic items would be owned only by those better off. From the initial 15-asset ownership questions, 10 were found to meet the requirements of a Mokken scale: (i) all were related to the same trait (wealth), (ii) ownership of one was independent of

This article has supplementary material available from erj.ersjournals.com

Received: Sept 232016 | Accepted after revision: Feb 282017

Support statement: Funding information for this article has been deposited with the Crossref Funder Registry. Funding was provided by the Wellcome Trust $(085790 / \mathrm{Z} / 08 / \mathrm{Z})$, with further local funding from the following. Ile-Ife, Nigeria: The Wellcome Trust (08405/2/09/Z), National Population Commission, Ile-Ife, Osun State, Nigeria. Naryn and Chui, Kyrgyzstan: Kyrgyz Thoracic Society. Tirana, Albania: GlaxoSmithKline. Blantyre, Malawi: GlaxoSmithKline, Liverpool School of Tropical Medicine, the Malawi Liverpool Wellcome Trust. Riyadh, Saudi Arabia: The Saudi Thoracic Society, King Abdullah International Medical Research Center KAIMRC. Annaba, Algeria: Boehringer Ingelheim. Penang, Malaysia: GlaxoSmithKline Pharmaceutical Sdn. Bhd. Sousse, Tunisia: Boehringer Ingelheim. Fes, Morocco: Boehringer Ingelheim. Srinagar, India: Sher-i-Kashmir Institute of Medical Sciences, Srinagar, J\&K. Colombo, Sri Lanka: The Respiratory Diseases Study Group of Sri Lanka and the World Health Organisation - Sri Lanka.

Conflict of interest: Disclosures can be found alongside this article at erj.ersjournals.com 
ownership of the others, (iii) the probability of ownership increased monotonically with the trait being measured. The 10 selected assets in decreasing order of prevalence of ownership were electricity, television, cell phone, refrigerator, indoor bath or shower, indoor tap, flush toilet, washing machine, car and landline telephone. The scores of individuals were calculated by adding up the total number of these assets owned by the respondent or their household; the scores were therefore in the range 0 (no assets) to 10 (all assets). Previous work showed that the number of assets owned was correlated with educational level and other variables likely to be related to socioeconomic position, and the mean number of assets owned in each country was highly correlated with the GNI per capita of the particular country being surveyed [12]. We therefore referred to these scores as "wealth scores", as they are broadly indicative of an individual's wealth. We previously showed that the rank ordering of prevalence of ownership of these assets was fairly consistent across a wide range of countries with GNI/capita ranging from $\$ 4750$ to $\$ 53760$ (Cronbach's alpha=0.96), suggesting that this is a useful scale to compare individuals both within and between countries [12].

The association of the wealth score with spirometric outcomes was estimated for each of the sites using linear regression of the $\mathrm{FEV} 1 / \mathrm{FVC}$ ratio (expressed as a \%) on wealth score, with higher ratios indicating less airflow obstruction. The analysis was adjusted for age and sex. In a secondary analysis, we used logistic regression to evaluate the association between the wealth score and airflow obstruction as a binary outcome, defined as a FEV1/FVC ratio below the LLN. To be consistent with earlier BOLD publications we computed these values from reference values provided by the National Health and Nutrition Examination Survey (NHANES) III study for Caucasian Americans [15]. Since age and sex are used to determine the LLN for an individual, the logistic regression model for the odds ratio (OR) of obstruction was not adjusted further for these variables. All regressions were weighted to allow different probabilities of selection of the participants in the samples, and standard errors were adjusted for clustering or stratification included in the study design in each site, using survey analysis methods [9].

To investigate the extent to which any other factors could explain the association between wealth and chronic airflow obstruction, whether as confounders or as factors on a causal pathway between wealth score and FEV1/FVC, the regression analyses were repeated adjusting for factors previously identified as having an effect on the FEV1/FVC ratio or obstruction [9], and possibly associated with poverty including: body mass index (BMI), education, smoking, passive smoking, exposure to a dusty job, family history of breathing problems, childhood hospitalisation for breathing problems and previous diagnosis of TB [9]. Education was defined as the highest completed level of education on a six-point scale from none to completed university education, and modelled as a continuous variable assuming a linear trend in obstruction with increasing level of schooling. Smoking was defined as the respondent's lifetime exposure to cigarette smoking or to other tobacco products measured in equivalent pack-years, where one pack-year equalled twenty cigarettes per day for 1 year [16]. Exposure to a dusty job was the self-reported number of years for which the respondent had worked in a dusty job. The BMI was included in the models as a categorical variable as follows: $<18.5$ (underweight); $\geqslant 18.5$ and $<25$ (normal); $\geqslant 25$ and $\leqslant 30$ (overweight); or $>30$ (obese). The other potential confounding and explanatory factors were included as binary variables.

Pooled estimates of the associations of the wealth score with FEV1/FVC ratio and obstruction across sites were obtained with inverse variance-weighted meta-analysis, using random-effects models to allow for some heterogeneity between sites. Between-site heterogeneity was evaluated using the $\mathrm{I}^{2}$ statistic and the Q-test.

Subsequent to the main analysis, we also adjusted for current smoking status as a three category variable (current, former, or never-smoker), in addition to total pack-years, and exposure to open fires for cooking and/or heating for more than 20 years, neither of which had been independently associated with obstruction in the earlier analysis, and ever having suffered from asthma which, though associated with obstruction, was viewed as a diagnostic label rather than as a truly independent risk factor.

Finally, the relationship between poverty and FEV1/FVC ratio at an aggregate level in the 12 sites was investigated by plotting the mean FEV1/FVC against the mean wealth score for each site. The percentage of variation in mean FEV1/FVC explained by the mean wealth score $\left(\mathrm{r}^{2}\right.$ value) in the 12 sites was estimated using linear regression, weighted for the number of subjects in each site. The relationship between mean wealth score and percentage of subjects with airflow obstruction (FEV1/FVC $<$ LLN) in each site was similarly analysed.

All analyses were carried out using the Stata v13.1 software (StataCorp, College Station, TX, USA).

Ethics

The study was approved by the local ethical committee in each site and by the Charing Cross Ethical Committee in London (06/Q0411/97), the relevant committee for the co-ordinating centre. Participants were only included if they gave written informed consent. 
Role of the funding source

The funders played no part in the design or implementation of this study nor in the decision to publish the results.

\section{Results}

Data were collected from 12 sites in 11 countries. Each site collected useable information from between 400 and 1100 individuals that was fairly evenly divided between men and women. Response rates were mostly over 70\%, but ranged from 46\% (Chui, Kyrgyzstan) to 92\% (Annaba, Algeria) (table 1). Overall, of 13903 people surveyed, 9255 provided adequate information and were included in the analyses. The centres included in this study were those that had information on the wealth score. They were biased towards the poorer countries, but the mean wealth score ranged from 4 in Blantyre (Malawi) and Kashmir (India) to 10 in Riyadh (Saudi Arabia). Smoking was light to moderate in all sites, except Kashmir. The high number of pack-years observed in Kashmir was due to two factors. Firstly, in Kashmir, heavy smoking is prevalent among the older population, in contrast to most other areas, where smoking is heaviest among the younger cohorts. Secondly, the most common type of smoking in Kashmir requires the use of the shisha pipe, which is given a relatively high number of equivalent pack-years compared to cigarettes in the formula of MASTERS and TUTT [16]. In most other areas, smoking is heaviest among the younger cohorts. The overall mean age was 53 (table 1). Consistent with these findings, the prevalence of obstruction (FEV1/FVC $<\mathrm{LLN})$ was generally low, with the lowest prevalence in Riyadh and Penang (Malaysia) (3\%), and the highest in Kashmir (16\%).

Regression analysis adjusted for age and sex showed that FEV1/FVC(\%) was significantly associated with the wealth score, increasing on average by $0.36 \%$ (absolute change) for each point increase in the score (95\% CI: 0.22-0.49; $\mathrm{p}<0.001$ ), and was higher in women and reduced with increasing age (table 2). When adjusted for other potential confounders, a major change in the association between airway obstruction and sex was noted, due to the adjustment for the confounding effects of smoking. However, a strong association between $\mathrm{FEV}_{1} / \mathrm{FVC}(\%)$ and wealth was still evident, with an increase of $0.23 \%$ per point increase in the score (95\% CI: $0.11-0.34 ; \mathrm{p}<0.001)$. In addition, the effects of age and sex, lower BMI, smoking, occupational exposure to dust and a history of tuberculosis were associated with lower FEV1/ FVC values (table 2). However, none of these individual factors had a strong confounding effect on the association between wealth score and $\mathrm{FEV}$ /FVC ratio. Among the dusty jobs, the most common was farming. When the effect of wealth score on the FEV1/FVC ratio was further adjusted for asthma, current smoking status and whether or not the individual had been exposed to $>20$ years of using open fires for cooking and/or heating (table S1) no appreciable change in the coefficient for the wealth score $(0.20)(95 \%$ CI: $0.08,0.33 ; \mathrm{p}=0.002)$ ) was evident.

Figure 1 shows that there was no significant heterogeneity between individual sites in the association between the FEV1/FVC and wealth score $\left(\mathrm{I}^{2}<1 \%\right.$; $\left.\mathrm{p}_{\text {het }}=0.44\right)$. The two estimates that were most against the trend were from the two richest countries, Saudi Arabia and Malaysia.

When the prevalence of obstruction $\left(\mathrm{FEV}_{1} / \mathrm{FVC}<\mathrm{LLN}\right)$ was regressed against the wealth score (adjusted for potential confounders), the prevalence fell by approximately $9 \%$ (relative change) for each point increase in the wealth score (OR: 0.91; 95\% CI: 0.84-0.98; $\mathrm{p}=0.010)$. Whilst there was evidence of some heterogeneity between sites, this was not statistically significant $\left(I^{2}=17 \%\right.$; $\left.\mathrm{p}_{\text {het }}=0.275\right)$ (figure 2 ).

Figure $3 \mathrm{a}$ shows the same positive association between FEV1/FVC ratio and wealth score at the aggregate level, as was found within sites. Approximately $40 \%$ of the variation in mean FEV1/FVC ratio could be explained by variations in the mean wealth score $\left(\mathrm{r}^{2}=0.385, \mathrm{p}=0.031\right)$. A corresponding trend was observed for the proportion of subjects with airflow obstruction among the different sites. This proportion showed a decline as the mean wealth score increased $\left(\mathrm{r}^{2}=0.358, \mathrm{p}=0.040\right)$ (figure $\left.3 \mathrm{~b}\right)$.

\section{Discussion}

Our study demonstrates an increase in FEV1/FVC and a reduction in the risk of airflow obstruction with increasing wealth, both within and across the 12 BOLD sites located in 11 countries.

These findings are consistent with the generally reported association between poverty and poor lung function [6], although much of the previous literature is related to the $\mathrm{FEV}$, which can reflect either reduced lung volume or reduced airflow with a normal lung volume, rather than the FEV $1 / \mathrm{FVC}$ ratio, which provides a more appropriate measure of airflow obstruction. The present findings are also consistent with the association between COPD mortality and GNI at an ecological level [4]. They are less obviously consistent with an earlier report from the BOLD study [3], which found little relationship between GNI and the prevalence of airflow obstruction, and a much stronger relationship between a low FVC and GNI. Only two of the sites in the earlier analysis, Sousse (Tunisia) and Kashmir (India), were also included in 
TABLE 1 Characteristics of the study population in the 12 centres $^{\#}$

\begin{tabular}{|c|c|c|c|c|c|c|c|c|c|c|c|c|}
\hline & $\begin{array}{l}\text { Sousse, } \\
\text { Tunisia }\end{array}$ & $\begin{array}{l}\text { Kashmir, } \\
\text { India }\end{array}$ & $\begin{array}{c}\text { Ife, } \\
\text { Nigeria }\end{array}$ & $\begin{array}{c}\text { Fes, } \\
\text { Morocco }\end{array}$ & $\begin{array}{l}\text { Tirana, } \\
\text { Albania }\end{array}$ & $\begin{array}{l}\text { Annaba, } \\
\text { Algeria }\end{array}$ & $\begin{array}{l}\text { Penang, } \\
\text { Malaysia }\end{array}$ & $\begin{array}{l}\text { Riyadh, } \\
\text { S. Arabia }\end{array}$ & $\begin{array}{c}\text { Blantyre, } \\
\text { Malawi }\end{array}$ & $\begin{array}{c}\text { Sri } \\
\text { Lanka }\end{array}$ & $\begin{array}{c}\text { Naryn, } \\
\text { Kyrgyzstan }\end{array}$ & $\begin{array}{c}\text { Chui, } \\
\text { Kyrgyzstan }\end{array}$ \\
\hline Subjects $n^{\pi}$ & 798 & 1077 & 1683 & 974 & 1188 & 938 & 1200 & 843 & 758 & 1408 & 1160 & 1876 \\
\hline Participants n $(\%)^{+}$ & $660(83)$ & 738 (69) & $864(51)$ & 760 (78) & 928 (78) & 862 (92) & $649(54)$ & $679(81)$ & 402 (53) & $1035(74)$ & $820(71)$ & $858(46)$ \\
\hline Males & 51 & 52 & 54 & 52 & 49 & 50 & 49 & 52 & 54 & 48 & 48 & 42 \\
\hline Age years & $52 \pm 9$ & $52 \pm 10$ & $53 \pm 11$ & $55 \pm 12$ & $56 \pm 12$ & $54 \pm 11$ & $55 \pm 10$ & $51 \pm 8$ & $51 \pm 9$ & $53 \pm 9$ & $52 \pm 10$ & $53 \pm 10$ \\
\hline$B M I \geqslant 25$ & 76 & 23 & 44 & 66 & 73 & 69 & 55 & 88 & 40 & 45 & 62 & 65 \\
\hline Height $\mathrm{cm}$ & $164 \pm 9$ & $160 \pm 9$ & $165 \pm 8$ & $162 \pm 9$ & $164 \pm 9$ & $164 \pm 10$ & $159 \pm 8$ & $162 \pm 9$ & $163 \pm 8$ & $157 \pm 9$ & $162 \pm 9$ & $163 \pm 9$ \\
\hline Smoking pack years ${ }^{\S}$ & $16 \pm 25$ & $133 \pm 207$ & $1 \pm 6$ & $8 \pm 19$ & $12 \pm 19$ & $11 \pm 19$ & $6 \pm 16$ & $9 \pm 24$ & $2 \pm 26$ & $3 \pm 9$ & $6 \pm 12$ & $10 \pm 18$ \\
\hline Passive smoking $f$ & 35 & 64 & 2 & 12 & 38 & 11 & 26 & 6 & 3 & 8 & 3 & 11 \\
\hline Dusty job"\#\# & 50 & 1 & 38 & 44 & 64 & 28 & 32 & 17 & 26 & 36 & 6 & 34 \\
\hline Tuberculosis & 0 & 0 & 1 & 1 & 1 & 2 & 0 & 2 & 5 & 1 & 1 & 1 \\
\hline $\begin{array}{l}\text { Family history of } \\
\text { COPD }\end{array}$ & 6 & 2 & 0 & 10 & 8 & 6 & 5 & 3 & 20 & 3 & 2 & 7 \\
\hline $\begin{array}{l}\text { Childhood } \\
\text { hospitalisation } \$ \S \S\end{array}$ & 3 & 0 & 1 & 1 & 1 & 2 & 3 & 1 & 1 & 3 & 1 & 7 \\
\hline $\begin{array}{l}\text { Post primary } \\
\text { education }^{f f}\end{array}$ & 50 & 18 & 59 & 28 & 80 & 56 & 65 & 65 & 46 & 73 & 94 & 97 \\
\hline FEV 1 FVC $\%$ & $80 \pm 7$ & $77 \pm 10$ & $79 \pm 8$ & $79 \pm 8$ & $78 \pm 10$ & $78 \pm 7$ & $81 \pm 7$ & $83 \pm 6$ & $79 \pm 8$ & $80 \pm 9$ & $78 \pm 7$ & $76 \pm 9$ \\
\hline$F_{E V} / F V C<L L N$ & 5 & 16 & 7 & 9 & 9 & 7 & 3 & 3 & 7 & 8 & 8 & 13 \\
\hline Wealth score ${ }^{\# \# \#}$ & $9 \pm 1$ & $4 \pm 2$ & $5 \pm 2$ & $8 \pm 2$ & $9 \pm 2$ & $8 \pm 1$ & $9 \pm 1$ & $10 \pm 0$ & $4 \pm 3$ & $6 \pm 2$ & $5 \pm 1$ & $6 \pm 2$ \\
\hline $\begin{array}{l}\text { GNI per capita US\$ } \\
\text { PPP }\end{array}$ & 9680 & 4750 & 4930 & 6160 & 9950 & 12860 & 22530 & 53760 & 750 & 9480 & 3100 & 3100 \\
\hline Asthma ever & 8 & 2 & 1 & 7 & 6 & 8 & 4 & 31 & 5 & 6 & 2 & 3 \\
\hline $\begin{array}{l}\text { Open fire } \\
>20 \text { years }\end{array}$ & 23 & 100 & 27 & 22 & 91 & 18 & 26 & 17 & 51 & 44 & 88 & 55 \\
\hline Smoker $^{+++}$ & 45 & 53 & 14 & 30 & 37 & 39 & 25 & 26 & 17 & 24 & 31 & 38 \\
\hline
\end{tabular}

Data are presented as mean \pm SD or \%, unless otherwise stated. BMI: body mass index; COPD: chronic obstructive pulmonary disease; FEV1: forced expiratory volume in 1 s; FVC: forced vital capacity: LLN : lower limit of normal. GNI: gross national income; PPP: purchasing power parity. \#. individual responses have been weighted according to the probability of selection to provide population estimates of means and percentages for each of the study areas; ${ }^{\text {? }}$ : individuals selected for possible inclusion in the Burden of Obstructive Lung Disease (BOLD) study using a random sampling process; ${ }^{+}$: individuals included in the study (i.e. those who agreed to take part in the BOLD study and provided useable data from both the questionnaire and spirometry tests); ${ }^{\S}: 1$ pack-year equals an average of 20 cigarettes per day for 1 year or the equivalent amount of other types of tobacco smoking; ${ }^{f}$ : somebody else in the household smoked during the past 2 weeks; ${ }^{\# \#}$ : the respondent was employed in a dusty job for at least 1 year; ${ }^{\text {} 11}$ : ever diagnosed with tuberculosis; ${ }^{++}$: a close sibling or parent had been diagnosed as having emphysema, chronic bronchitis or COPD by a health professional; $\$ \$$ : hospitalised for breathing problems before the age of 10 years; ${ }^{f f}$ : highest level of education completed

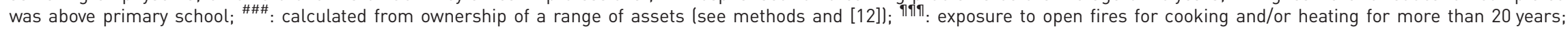
was above primary school;
+++ : current or ex-smoker. 
TABLE 2 Associations between forced expiratory volume in $1 \mathrm{~s}$ (FEV1)/forced vital capacity (FVC) \% and wealth score, adjusted for age and sex only and then for multiple potential confounding factors

\begin{tabular}{|c|c|c|c|c|c|c|}
\hline & \multicolumn{3}{|c|}{ Adjusted for age and sex only } & \multicolumn{3}{|c|}{$\begin{array}{l}\text { Adjusted for age, sex and other } \\
\text { potential confounders }\end{array}$} \\
\hline & Coefficient & $95 \% \mathrm{Cl}$ & p-value & Coefficient & $95 \% \mathrm{Cl}$ & p-value \\
\hline Wealth Score & 0.36 & $0.22-0.49$ & $<0.001$ & 0.23 & $0.11-0.34$ & $<0.001$ \\
\hline Females & 2.43 & $1.63-3.24$ & $<0.001$ & 0.76 & $0.01-1.50$ & 0.046 \\
\hline Age years & -0.27 & $-0.31--0.23$ & $<0.001$ & -0.25 & $-0.29--0.22$ & $<0.001$ \\
\hline \multicolumn{7}{|l|}{ BMI } \\
\hline$<18.5$ & & & & -0.85 & $-2.64-0.94$ & 0.351 \\
\hline$\geqslant 18.5-<25$ & & & & 0 & & \\
\hline$\geqslant 25-\leqslant 30$ & & & & 0.85 & $0.50-1.19$ & $<0.001$ \\
\hline$>30$ & & & & 1.23 & $0.68-1.77$ & $<0.001$ \\
\hline Education level completed & & & & -0.10 & $-0.32-0.12$ & 0.383 \\
\hline Smoking pack-years & & & & -0.06 & $-0.09--0.04$ & $<0.001$ \\
\hline Passive smoking & & & & -0.31 & $-1.14-0.53$ & 0.474 \\
\hline Worked in a dusty job years & & & & -0.04 & $-0.06--0.01$ & 0.003 \\
\hline Family history of COPD & & & & -0.75 & $-1.87-0.37$ & 0.191 \\
\hline Hospitalised as a child & & & & -2.44 & $-5.18-0.29$ & 0.080 \\
\hline Tuberculosis & & & & -2.30 & $-4.40--0.19$ & 0.032 \\
\hline
\end{tabular}

Coefficients indicate the effect of an individual having the stated characteristic lversus not having that characteristic) on $\mathrm{FEV}_{1} / \mathrm{FVC} \%$ or for a one-unit increase in that variable, adjusted for all other variables in that column le.g. individuals with a history of tuberculosis had an $\mathrm{FEV}_{1} / \mathrm{FVC} 2.3 \%$ lower on average than comparable individuals without a history of tuberculosis. Bold denotes statistical significance. COPD: chronic obstructive pulmonary disease.

the present analysis, as the questions on household assets were only introduced into the BOLD protocol at a relatively late stage. Thus, the wealth scores could not be calculated for the earlier sites. One possible reason for the inconsistency between our results and previous ecological evidence from the BOLD study is the low to medium levels of smoking rates in almost all of the sites analysed in the present study, with the exception of Kashmir. Smoking is by far the most important cause of airflow obstruction [9], and the relationship between income and smoking is complicated. Where income is very low, smoking is usually

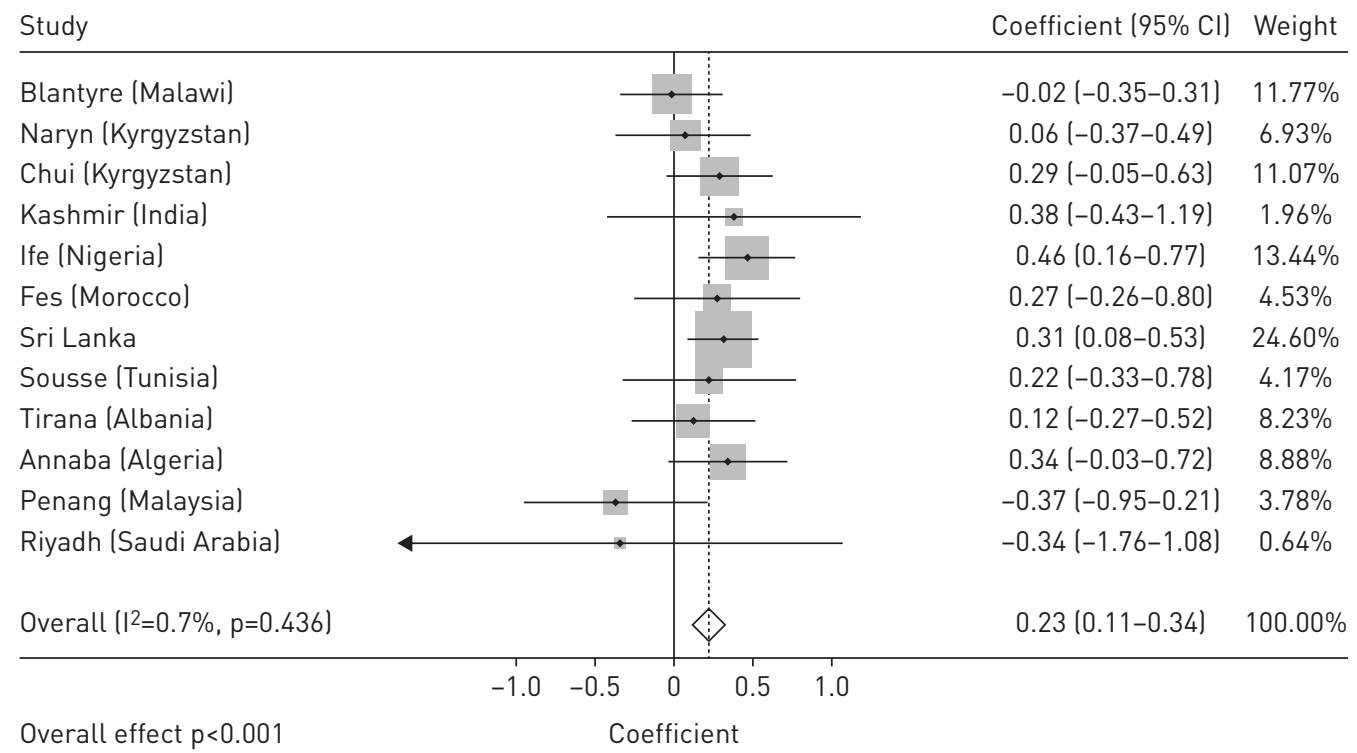

FIGURE 1 Forest plot for the effect of wealth score on forced expiratory volume in $1 \mathrm{~s}$ (FEV 1 )/forced vital capacity (FVC), adjusted for age, sex and all other confounders (body mass index, education, smoking, passive smoking, exposure to a dusty job, family history of breathing problems, childhood hospitalisation for breathing problems and previous diagnosis of tuberculosis). The coefficient gives the mean change in FEV $1 / F V C$ (\%) for a one-unit increase in the wealth score in each of the sites. 


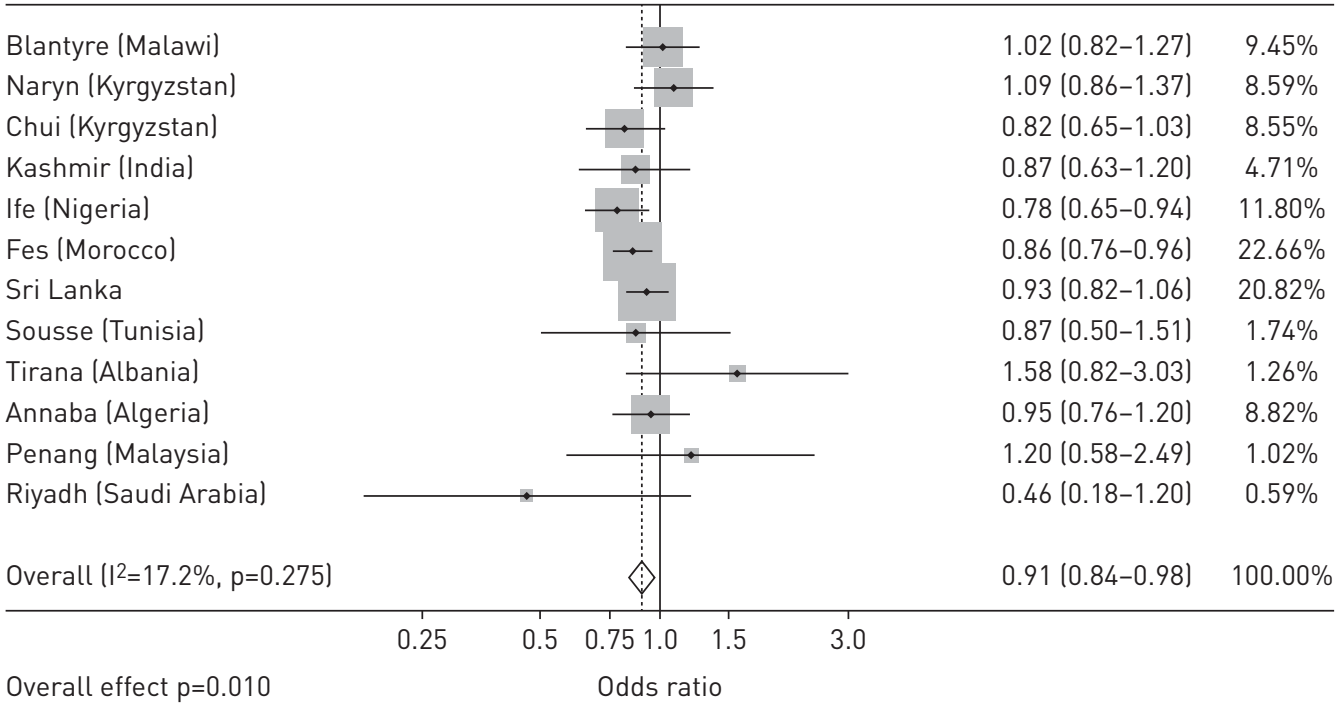

FIGURE 2 Forest plot showing the association between the odds of having forced expiratory volume in $1 \mathrm{~s} /$ forced vital capacity below the lower limit of normal (based on The National Health and Nutrition Examination Survey (NHANES) equations) and wealth score. The odds ratios show the effect of a one-unit increase in the wealth score on the odds of obstruction in each of the sites, adjusted for body mass index, education, smoking, passive smoking, working in a dusty job, family history of chronic obstructive pulmonary disease, hospitalisation as a child for breathing problems and tuberculosis.

rare. As income rises, smoking also increases; however, in affluent countries, smoking is predominantly a habit of the less well off.

The mechanisms by which poverty is associated with airflow obstruction are unknown. In the present analysis, the strongest confounder was age, which was negatively associated with both FEV1/FVC and wealth. However, some of this association was also explained by associations with a lower BMI, a history of tuberculosis, or of working in a dusty job in addition to smoking. In a cross-sectional study, it is difficult to ascertain to what extent these variables should be viewed as confounders, and as mediating variables on a causal pathway between poverty and low lung function. Even after adjustment for potential confounding and explanatory variables measured in the present study, there was a consistent inverse association between the risk of airflow obstruction and the wealth score, which appears to explain $35 \%$ of the variation in the prevalence of a low FEV1/FVC ratio. The explanation for this association is not simple. The association between poverty and airflow obstruction among individuals in each site could be explained
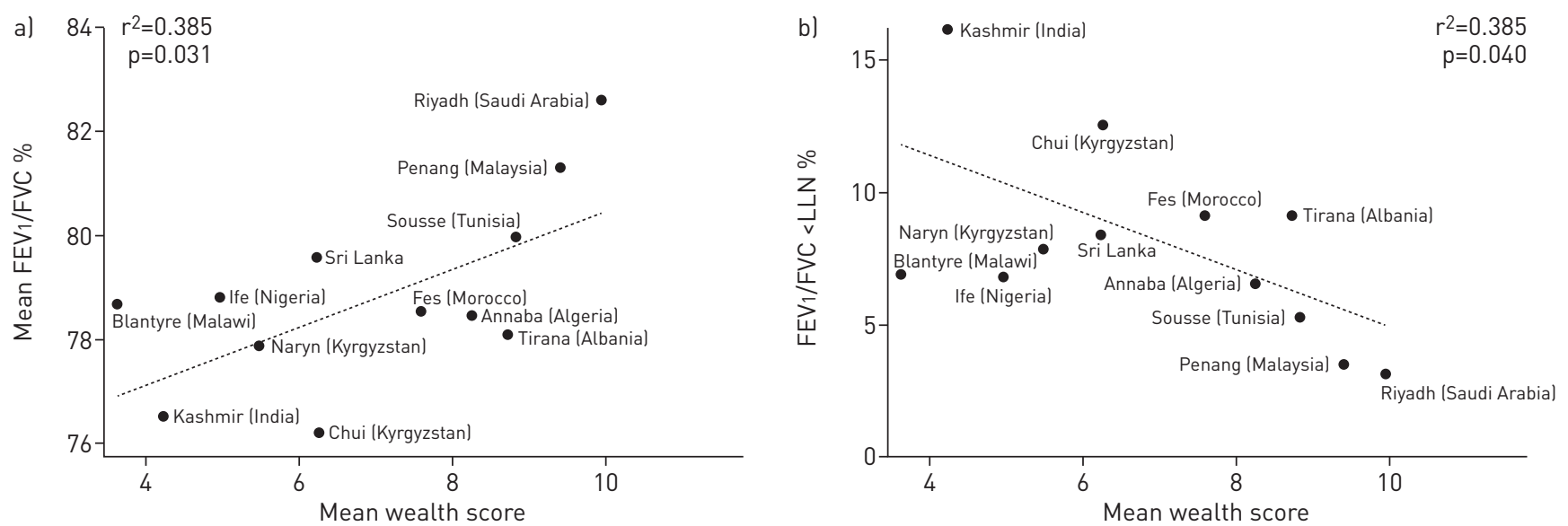

FIGURE 3 Association between site-level means of wealth score and a) mean forced expiratory volume in $1 \mathrm{~s}(\mathrm{FEV}$ ) $) /$ forced vital capacity (FVC) (\%), b) proportion of subjects with obstruction ( $\mathrm{FEV}_{1} / \mathrm{FVC}<$ lower limit of normal). The dashed lines show the linear regression lines of best fit weighted to allow for the number of subjects included in each site. 
by unmeasured confounders, or by error in measured confounders. However, the consistency of findings across sites and the association between poverty and obstruction observed at the aggregate level among the 12 sites suggest that poverty is an important explanatory variable requiring further investigation, and a potentially important policy target when addressing poor lung health.

BARKER and OSMOND [17] pointed out the strong ecological association between high mortality from pneumonia and bronchitis in early life and mortality from chronic lung disease half a century later among adults born at the same time, and BARKER et al. [18] subsequently showed a relationship between birth weight and weight at 1 year and both mortality from COPD and low lung function. They also pointed out the strong association between poverty and both outcomes. However, the association of birth weight and weight at 1 year was mainly evident with FVC rather than FEV1/FVC. Diet is possibly a more likely explanation for that association. Although the nature of the association between diet and lung function remains unclear, an association exists [19] and is likely influenced by household resources. On the other hand, much of the association with diet was found only among smokers, whilst tobacco consumption was low in the sample examined in the current analysis.

The BOLD study is a large multinational study of ventilatory function and risk factors of chronic lung disease. The central standardised protocol and training programme ensured consistency across the sites, and the quality of the spirometry was strictly controlled by central checks on all tests. The sites represented in the present analysis are very diverse in wealth, culture and social conditions. The consistency of the findings across these sites supports the finding of a strong relationship between poverty and level of airflow obstruction.

The wealth score used is based on household assets, which are easy to count and conveniently form a one-dimensional score, the average of which correlates well with the per capita GNI of the countries in which it was evaluated [12]. However, wealth is not a one-dimensional concept and an understanding of the relationship between this measure and airway obstruction requires further study. Moreover, this instrument is likely to be more sensitive at detecting differences in poorer countries [12], although this is probably not a substantial problem in this case, as the evidence from mortality rates strongly suggests that the association with poverty is predominantly seen among countries with a per capita GNI below approximately US\$ 20000 per annum. Our analysis is limited to the effects on chronic airflow obstruction and does not consider the effects on vital capacity. This is because the standardisation of the FEV1/FVC ratio using a single norm is less controversial than standardisation of the FVC [15]. With only 12 sites in 11 countries, this would have been impossible.

The present study has a number of limitations. First, although the sites covered a broad range of socioeconomic conditions, they are not entirely representative of the global range, and the present analysis included only 12 BOLD sites. This lack of complete geographical representation could potentially produce results that might not otherwise be reproduced in a more comprehensive study or might show weaker associations. Second, our study was unable to identify intermediate variables that adequately explained the observed associations. This might be in part, because some of the important variables had not been collected in our study, or because the variables had been measured with low precision or accuracy. There is therefore a need to investigate further the role of factors that can explain why poverty is associated with airway obstruction. This limitation is likely to be more important in a study that relies heavily on self-reported exposures.

In conclusion, in countries with a low overall consumption of tobacco, our study revealed an inverse association between wealth and airflow obstruction (a low FEV1/FVC ratio). Sites with a relatively high mean wealth score had a relatively low prevalence of airflow obstruction. Understanding this relationship better might provide the necessary solutions to the improvement of lung health in poorer countries, particularly those where smoking is still uncommon.

\section{Acknowledgements}

Author contributions: P.G.J. Burney, C. Minelli and J. Townend designed the analysis and drafted the initial manuscript. J. Townend and J. Patel carried out statistical analysis. K. Mortimer, D.O. Obaseki, M. Al Ghobain, H. Cherkaski, M. Denguezli, K. Gunesekera, H. Hafizi, P.A. Koul, L.C. Loh, C. Nejjari, T. Sooronbayev and S.A. Buist contributed to the design and conduct of the study. All authors critically reviewed and approved the final manuscript.

\section{References}

1 Lozano R, Naghavi M, Foreman K, et al. Global and regional mortality from 235 causes of death for 20 age groups in 1990 and 2010: a systematic analysis for the Global Burden of Disease Study 2010. Lancet 2013; 380: 2095-2128.

2 Vos T, Flaxman AD, Naghavi M, et al. Years lived with disability (YLDs) for 1160 sequelae of 289 diseases and injuries 1990-2010: a systematic analysis for the Global Burden of Disease Study 2010. Lancet 2012; 380: 2163-2196. 
3 Burney P, Jithoo A, Kato B, et al. Chronic obstructive pulmonary disease mortality and prevalence: the associations with smoking and poverty - a BOLD analysis. Thorax 2014; 69: 465-473.

4 Burney P, Patel J, Newson R, et al. Global and regional trends in COPD mortality, 1990-2010. Eur Respir J 2015; 45: 1239-1247.

5 Office of Population Census and Surveys. Occupational Mortality: the Registrar General's Decennial Supplement for Great Britain, 1979-80, 1982-83, Great Britain. London, H.M. Stationery Office, 1986.

6 Hegewald M, Crapo R. Socioeconomic status and lung function. Chest 2007; 132: 1608-1614.

7 Krzyzanowski M, Kauffmann F. The relation of respiratory symptoms and ventilatory function to moderate occupation exposure in a general population. Int J Epidemiol 1988; 17: 397-399.

8 Demissie K, Ernst P, Hanley JA, et al. Socioeconomic status and lung function among primary school children in Canada. Am J Respir Crit Care Med 1996; 153: 719-723.

9 Hooper R, Burney P, Vollmer W, et al. Risk factors for COPD spirometrically defined from the lower limit of normal in the BOLD project. Eur Respir J 2012; 39: 1343-1353.

10 Obaseki DO, Erhabor GE, Gnatiuc L, et al. Chronic airflow obstruction in a black African population: results of BOLD study, Ile-Ife, Nigeria. COPD: J Chron Obstruct Pulmon Dis 2016; 13: 42-49.

11 Buist AS, Vollmer WM, Sullivan SD, et al. The Burden of Obstructive Lung Disease Initiative (BOLD): rationale and design. COPD: J Chron Obstruct Pulmon Dis 2005; 2: 277-283.

12 Townend J, Minelli C, Harrabi I, et al. Development of an international scale of socio-economic position based on household assets. Emerg Themes Epidemiol 2015; 12: 13.

13 Mokken RJ. A Theory and Procedure for Scale Analysis: With Applications in Political Research. Berlin, de Gruyter, 1971.

14 Hardouin J, Bonnaud-Antignac A, Bille V. Nonparametric item response theory using Stata. Stata J 2011; 11: $30-51$.

15 Hankinson JL, Odencrantz JR, Fedan KB. Spirometric reference values from a sample of the general U.S. population. Am J Respir Crit Care Med 1999; 159: 179-187.

16 Masters N, Tutt C. Smoking pack Year Calculator. 2007. http://smokingpackyears.com/ Date last accessed: September 18, 2015.

17 Barker DJP, Osmond C. Childhood respiratory infection and adult chronic bronchitis in England and Wales. Br Med J (Clin Res Ed) 1986; 293: 1271-1275.

18 Barker DJP, Godfrey KM, Fall C, et al. Relation of birth weight and childhood respiratory infection to adult lung function and death from chronic obstructive airways disease. BMJ 1991; 303: 671-675.

19 Shaheen SO, Jameson KA, Syddall HE, et al. The relationship of dietary patterns with adult lung function and COPD. Eur Respir J 2010; 36: 277-284. 